\title{
MIROSŁAW LOBA
}

Université Adam Mickiewicz, Poznań

\section{LE CORPS DE ROLAND BARTHES}

\begin{abstract}
Loba Mirosław, Le corps de Roland Barthes [Roland Barthes Body], Studia Romanica Posnaniensia, Adam Mickiewicz University Press, Poznań, vol. XXXVII/1: 2010, pp. 3-11. ISBN 978-83-2322145-6. ISSN 0137-2475. DOI 10.2478/v10123-010-0001-7.

The aim of this paper is to present the relationship between body and linguistic and literary signs in the work of Roland Barthes. The main problem is Roland Barthes thinking on semiotic representation of the body as an ambivalent process of selfconstruction and alienation.
\end{abstract}

Envisager les rapports entre le corps et le signe dans la pensée de Roland Barthes, c'est ouvrir d'emblée deux perspectives : la première qui se réfère à la réflexion sur le corps et sa représentation sémiotique en général, la seconde qui porte sur l'expérience du corps à travers les signes et les images. Vu l'ampleur du problème je me limiterai à la deuxième approche. Mais en fait, est-il possible de les séparer définitivement ? Les deux réflexions ne se rencontrent-elles pas inévitablement et ne s'éclairent-elles pas réciproquement? C'est ainsi que le lecteur est invité à lire Roland Barthes par Roland Barthes : «On ne trouvera donc ici, mêlées au roman familial, que les fulgurations d'une préhistoire du corps qui s'achemine vers le travail, la jouissance d'écriture » (Barthes, 1975, p. 6).

En abordant l'œuvre et la vie de Roland Barthes tout porte à croire que l'écrivain est celui à qui le corps fait problème. L'expérience juvénile du corps malade l'avait tellement marqué qu'il y revient constamment dans ses écrits postérieurs. La tuberculose qui l'avait frappé dans sa jeunesse lui a fait voir que le corps était le lieu où prennent naissance des discours qui dénaturaient le vécu, que le destin du corps était inévitablement sémiotique et que le sentiment d'avoir un corps n'avait rien de naturel. Comme le dit Ivan Almeida :

Toute entrée dans le monde des signes implique un assujettissement aux fictions de la représentation institutionnelle, à commencer par celle du langage, qui ne peut pas nommer sans classifier et différer (Almeida, 1983, p. 11).

Á la mise en scène fragmentaire de sa vie, Barthes donnera un commentaire inscrit sur la couverture du livre : «Tout ceci doit être considéré comme dit par un personnage 
de roman » (Barthes, 1975). À partir de cette découverte de l'aliénation corporelle, de l'inadéquation du corps et de ses représentations, l'existence sémiotique se fait un thème majeur des recherches et des écrits barthésiens. Il y revient de façon plus ou moins voilée dans chacun de ses livres. Suite à la maladie Barthes comprend que la représentation institutionnelle du corps non seulement ne permet pas d'y accéder directement, mais elle devient également un moyen d'exclusion. Les symptômes corporels perdent de leur opacité une fois entrés dans l'évidence des signes et des images de l'institution hospitalière, une fois pris en charge par le discours médical, bref par le langage. Son livre Roland Barthes par Roland Barthes se termine par une sorte d'aveu d'échec : "Écrire le corps. Ni la peau, ni les muscles, ni les os, ni les nerfs, mais le reste: un ça balourd, fibreux, pelucheux, effiloché, la houppelande d'un clown" (Barthes, 1975, p. 182). Or le corps transposé en image ou représenté dans le texte, le corps transfiguré en signe demeure, un ça, une pulsion au sens freudien, une énergie dont les manifestations sont loin de saisir le secret de sa force. Le ça est toujours mal représenté, il est atteint d'un malaise de la représentation ratée. L'interrogation qui obsède tout le travail barthésien est cette inadéquation entre le corps et sa figuration :

Ne croyant pas à la séparation de l'affect et du signe, de l'émotion et de son théâtre, il ne pouvait exprimer une admiration, une indignation, un amour, par peur de le signifier mal. Aussi, plus il était ému, plus il était terne. Sa « sérénité » n'était que la contrainte d'un acteur qui n'ose entrer en scène par crainte de trop mal jouer (Barthes, 1975, p. 180).

Quel corps? Nous en avons plusieurs.

La figuration opaque de la chair, voire la représentation sémiotique du corps, posent pour Barthes le problème de l'authenticité de l'expérience de son propre corps, de son unicité. S'il répète souvent que l'individu a plusieurs corps qui sont avant tout l'effet de la force classificatoire du langage, il n'arrête pas de croire à l'individualité de son corps, et il est presque prêt à adhérer avec étonnement à l'exaltation chrétienne de l'individu et le caractère irréductible de son corps :

C'est précisément, sans doute, que je n'ai pas le même corps qu'eux; mon corps ne peut pas se faire à la généralité, à la puissance de généralité qui est dans le langage. - N'est-ce pas là une vue individualiste ? Ne la trouve-t-on pas chez un chrétien - antihégélien notoire - tel que Kierkegaard ? (Barthes, 1975, p. 178).

L'écriture de Barthes révèle donc constamment ce double mouvement dans l'observation de son corps réel et du théâtre du corps offert par la langue. D'un côté, il développe la réflexion sur la spécificité de son corps, sur la proximité du vivant et du sémiotique, sur leur profonde interférence, et d'autre part il insiste sur son caractère symbolique, sur ses postures culturelles, sur ses représentations institutionnelles et textuelles qui l'aliènent, le dépossèdent de soi-même.

Il est nécessaire de s'arrêter un instant sur ce lien très étroit entre le signe et l'affect, sur cet enracinement du sémiotique dans le corporel qui décident du caractère inédit de chaque être. Dans Le degré zéro de l'écriture Roland Barthes évoque de façon très 
rapide mais très suggestive la proximité du biologique et du style, conception qu'il n'abandonnera jamais, même dans la période la plus « lacanienne » de son œuvre. Barthes rattache le style à la poussée de l'énergie contenue dans un corps : « Le style est proprement un phénomène d'ordre germinatif, il est la transmutation d'une Humeur » (Barthes, 1953 et 1972, p. 16). Il retrouve, comme le fera plus tard Julia Kristeva, le pulsionnel dans les formations symboliques : « le style n'est jamais que métaphore, c'est-à-dire équation entre l'intention littéraire et la structure charnelle de l'auteur »(Barthes, 1953 et 1972, p. 17). On peut donc supposer que Barthes admet depuis le début de sa carrière que le passage des signes par le corps amène leur profonde modification, en grande partie inconsciente et difficilement contrôlable. Dans Fragments d'un discours amoureux, il constate :

Je puis tout faire avec mon langage, mais non avec mon corps. Ce que je cache par mon langage, mon corps le dit. Je puis à mon gré modeler mon message, non ma voix. A ma voix, quoi qu'elle dise, l'autre reconnaîtra que «j'ai quelque chose ». Je suis menteur (par prétérition) non comédien (Barthes, 1977, p. 54).

En fait, on pourrait se demander si cette conviction n'est pas issue de la fascination du théâtral chez Barthes. La concrétisation, l'interprétation d'un rôle trouve sa meilleure illustration dans cette rencontre toujours surprenante de la parole et du corps d'un comédien. Malgré la proximité du langage et du corps, Barthes insiste constamment sur la dépossession de son propre corps qui s'effectue à travers le langage :

Dès que je produis, dès que j'écris, c'est le Texte lui-même qui me dépossède (heureusement) de ma durée narrative. Le Texte ne peut rien raconter; il emporte mon corps ailleurs, loin de ma personne imaginaire... (Barthes, 1975, p. 6).

L'écriture même si elle reste corollaire du corps, elle est aussi porteuse de la puissance structurelle et rhétorique du langage, capable de dominer, de coloniser le sujet parlant, de l'emporter au-delà de ses désirs et ses intentions authentiques. La dépossession effectuée par l'écriture est donc résultat de la violence de la dénomination, voire de pratiques culturelles et institutionnelles qui imposent au corps différentes formes et identités sociales :

J'ai un corps digestif, j'ai un corps nauséeux, un troisième migraineux, et ainsi de suite : sensuel, musculaire (la main de l'écrivain), humoral, et surtout : émotif : qui est ému, bougé, ou tassé ou exalté, ou apeuré, sans qu'il y paraisse rien. D'autre part, je suis captivé jusqu'à la fascination par le corps socialisé, le corps mythologique, le corps artificiel (celui des travestis japonais) et le corps prostitué (de l'acteur). Et en plus de ces corps publics (littéraires, écrits), j'ai, si je puis dire, deux corps locaux : un corps parisien (alerte, fatigué) et un corps campagnard (reposé, lourd) (Barthes, 1975, p. 65).

Tous ces corps font partie de l'existence sémiotique de Roland Barthes. Leur longue liste ne sert qu'à confirmer la force aliénante du langage et de la culture par le simple fait de capturer et nommer tout ce théâtre corporel. L'individu ne se retrouve pas dans les images et les signes qui prétendent à le représenter : 
Il supporte mal toute image de lui-même, souffre d'être nommé. Il considère que la perfection d'un rapport humain tient à cette vacance de l'image : abolir entre soi, de l'un à l'autre, les adjectifs ; un rapport qui s'adjective est du côté de l'image, du côté de la domination, de la mort (Barthes, 1975, p. 47).

Cette dépossession est vécue chez Barthes de façon mélancolique : nommer quelque chose, c'est faire mourir un mot contre les autres. Le rapport direct au corps n'est jamais possible, il est toujours différé, dénaturé. Le corps est toujours déjà colonisé par le langage. Sa perception passe inévitablement par les signes qui impriment sur lui des stigmates et des blessures. Retenons donc cette conception paradoxale du corps barthésien qui consiste dans la coexistence de deux conceptions : d'un côté le voisinage étroit du corps et du signe, le signe étant toujours succédané de l'affect et d'autre part l'aliénation du corps due à sa perception effectuée à travers les signes.

\section{PERCEPTION DU CORPS}

Après avoir dégagé cette dualité corporelle il est temps de passer à l'approche de la manifestation de la perception des sensations corporelles. Il est nécessaire de signaler que la perception du corps selon Barthes n'a rien de commun avec la démarche proustienne : « mon corps ne marche pas dans l'histoire de la madeleine, des pavés et des serviettes de Balbec» (Barthes, 1975, p. 139). Le langage est un filtre, une interface entre le corps et le monde : « Le langage est une peau: je frotte mon langage contre l'autre » (Barthes, 1977, p. 87). Ceci veut dire qu'il refuse la description et l'analyse de l'intensité des perceptions. Mais il ne rate jamais le moindre détail du paraître du corps. Barthes privilégie notamment l'ouïe, le regard et l'odorat, mais il se méfie de leur représentation sémiotique et linguistique. Les fréquentes sensations auditives se focalisent chez Barthes autour de la perception de la voix. Dès Le Degré zéro de l'écriture passant par le protagoniste de la nouvelle de Balzac jusqu'à l'analyse du phénomène amoureux, Barthes ausculte les voix des chanteurs $(S / Z)$, de ses amis et il discerne dans le phonologique, dans l'activité vocalique les traces du culturel et du symbolique :

Eu égard aux sons de la langue, l'écriture à haute voix n'est pas phonologique, mais phonétique ; son objectif n'est pas la clarté des messages, le théâtre des émotions ; ce qu'elle cherche (dans une perspective de jouissance), ce sont les incidents pulsionnels, c'est le langage tapissé de peau, un texte où l'on puisse entendre le grain du gosier, la patine des consonnes, la volupté des voyelles, toute une stéréophonie de la chair profonde : l'articulation du corps, de la langue, non celle du sens, du langage (Barthes, 1973, p. 89).

Les voix, les vocalises ne sont donc jamais asémantiques. Elles révèlent « la matérialité du corps parlant sa langue maternelle : peut-être la lettre, sûrement la signifiance » (Barthes, 1982, p. 238). À travers l'activité vocale s'établit le je entre le phéno-chant et le géno-chant, entre les formes codées de la voix et la volupté des sons. 
Une autre sensation celle de voir, comme l'ouie, fait appel à une opposition très importante entre dehors et dedans. Le corps est porteur des signes de l'extériorité sociale qui le divise, qui le fragmente et l'exclue. Mais il n'oublie jamais le caractère provisoire de cette extériorité qui est aussi passagère que la chair :

Voici ce que j'ai fait un jour de mon corps : A Leysin, en 1945, pour me faire un pneumothorax extrapleural, on m'enleva un morceau de côte, qu'on me restitua ensuite solennellement, troussé dans un peu de gaze médicale (les médecins, suisses, il est vrai, professaient ainsi que mon corps m'appartient, dans quelque état dépiécé qu'ils me le rendent : je suis propriétaire de mes os, dans la vie comme dans la mort. Je gardai longtemps dans un tiroir ce morceau de moi-même [...] Et puis, un jour, comprenant que la fonction de tout tiroir est d'adoucir, d'acclimater la mort des objets en les faisant passer pour une sorte d'endroit pieux [...] mais n'allant pas jusqu'à oser jeter ce bout de moi-même dans la poubelle commune de l'immeuble, je balançai la côtelette et sa gaze, du haut du balcon, comme si je dispersais romantiquement mes propres cendres, dans la rue Servandoni, où quelque chien dut venir les flairer (Barthes, 1975, pp. 65-66).

Regarder/se regarder est donc un des thèmes majeurs de ses lectures de la chair et du monde. Je n'ai pas besoin de rappeler et de rapporter cette analyse du regard dans La chambre claire. Barthes démontre constamment comment il est impossible de voir clair les choses ; la vue, la vision directe sont toujours brouillées par les écrans et les filtres de notre mémoire, de notre savoir, de notre ignorance, de nos désirs. Le corps photographié en fournit la preuve - sa représentation, sa figuration repousse toujours par son caractère réducteur - « je n'ai jamais ressemblé à cela » (Barthes, 1975, p. 40). L'analyse du regard posé sur les corps des autres se présente de façon la plus saisissante et la plus troublante dans Incidents, un livre très intime, recueils de flashs de mémoire, de pensées et d'observations évoquant son séjour au Maroc et des soirées parisiennes. Barthes y fait voir les corps des autres et même son propre corps à travers le prisme du désir, et même à travers quelque chose de plus bas, à savoir la pulsion. Comment la pulsion fait-elle apparaître le corps. Elle n'arrête pas de le réduire, de le fragmenter, de le fétichiser :

Plus tard dans la soirée, au Flore, non loin de notre table, un autre, angélique avec ses cheveux longs coupés d'une raie au milieu ; de temps en temps il me regarde ; m'attire sa chemise très blanche ouverte sur sa poitrine ; il lit Le Monde et boit du Ricard, je crois ; ne part pas, finit par me sourire; il a de grosses mains, qui démentent la douceur et la délicatesse du reste ; c'est de ses mains que j'induis le gigolo (Barthes, 1977, p. 87).

Barthes procède par la technique de l'étrangement d'un objet, par son isolement, comme s'il procédait à la réduction phénoménologique. Mais en même temps, il recourt à la sémiotique et fait voir le caractère culturel de son regard. Là où l'on voit le mieux la confrontation de l'apparition apparemment neutre d'une chose, d'un objet, d'une partie du corps avec leur appréciation apportée par le langage, c'est quand il oppose sa propre délicatesse, sa propre culture nourrie de Proust à la vulgarité, à la grossièreté du regard des jeunes marocains et des gigolos parisiens. Mais il pratique 
aussi une stratégie inverse, c'est lui qui nomme avec des mots très crus la brutalité de la pulsion :

Ce pays : où il est possible que les boy-scouts aient mauvais genre. J'en ramène trois à la ville : un peu déguenillés, cheveux longs, dépeignés, chapeau fantaisie en bataille, chapeau coquin et cependant fanion, badges, salut scout, phraséologie ( $"$ les scouts sont frères de tout le monde »). Cuisses nues, le " responsable » bandait, pendant que les autres chantaient des chansons scoutes tristes (histoire d'un orphelin) (Barthes, 1987, p. 60).

Les sensations dont est affecté le corps barthésien conduisent à deux expériences qui organisent de façon décisive son existence sémiotique : le travail et la jouissancesouffrance [« Mon corps ne m'existe que sous deux formes courantes: la migraine et la sensualité » (Barthes, 1975, p. 64)]. Ces trois états sont une forme d'habiter et de sentir son corps, ils se distinguent par leur intensité et leur puissance.

Le travail est une activité qui rattache le corps barthésien à la nature, le corps s'efface et s'intègre avec la nature, retrouve son authenticité :

ce 6 août, à la campagne, c'est le matin d'un jour splendide : soleil, chaleur, fleurs, silence, calme, rayonnement. Rien ne rôde, ni le désir, ni l'agression ; seul le travail est là, devant moi, comme une sorte d'être universel : tout est plein. Ce serait donc cela, la Nature ? Une absence... du reste? La Totalité ? (Barthes, 1975, p. 181).

Cette remarque me paraît très pertinente, le travail n'est pas une activité aliénante, loin de le déposséder, il le rattache au monde naturel, il le réconcilie avec la vie.

\section{JOUISSANCE ET MIGRAINE}

La jouissance embrasse plusieurs pratiques qui se distinguent l'une de l'autre par le degré de leur intensité : érotisme, plaisirs intellectuels, cuisine, musique. La jouissance que Barthes éprouve n'est pas un état psychique, n'est pas une extase qui porte le corps hors de lui. Au contraire, elle est enracinée dans son être charnel. La chair est simplement gaie et on pourrait même dire qu'elle est le lieu où le moi s'éprouve dans son ipséité :

J'aime, je n'aime pas : cela n'aucune importance ; cela, apparemment, n'a pas de sens. Et pourtant tout cela veut dire : mon corps n'est pas le même que le vôtre. Ainsi dans cette écume anarchique des goûts et des dégoûts, sorte de hachurage distrait, se dessine peu à peu la figure d'une énigme corporelle, appelant complicité ou irritation. Ici commence l'intimidation du corps, qui oblige l'autre à me supporter libéralement, à rester silencieux et courtois devant des jouissances ou des refus qu'il ne partage pas (Barthes, 1975, p. 121).

Mais d'autre part, la chair barthésienne est triste. Hélas ! (Et il a lu tous les livres.) La forme la plus fréquente que prend le corps souffrant est la migraine. « La migraine - écrit Barthes - n'est que le tout premier degré du mal physique et la sensualité n'est considérée ordinairement que comme une sorte de laissé-pour-compte de la jouissance 
» (Barthes, 1975, p. 64). Le faible théâtre du mal de tête se montre un mécanisme de défense comme il le dit lui-même :

En d'autres termes, mon corps n'est pas un héros. Le caractère léger, diffus, du mal ou du plaisir (la migraine elle aussi caresse certaines de mes journées) s'oppose à ce que le corps se constitue en lieu étranger, halluciné, siège de transgressions aiguës (Barthes, 1975, p. 65).

Cette souffrance physique revêt également un caractère culturel [« La division sociale passe par mon corps : mon corps lui-même est social ", (Barthes, 1975, p. 128)]. Cependant l'explication psychologique et sociologique n'épuise pas tout le potentiel symbolique de la migraine. Barthes va jusqu'à interpréter ce mal de tête comme l'expérience de l'opacité du corps. Dans ces derniers textes la migraine est aussi considérée comme l'entrée « dans la maladie mortelle de l'homme : la carence de symbolisation » (Barthes, 1975, p. 129). La migraine est donc le moment où le corps cherche à échapper à la fatalité de la signification où il se soustrait à la violence de la dénomination.

Cette dialectique de la jouissance et de la souffrance qui traverse l'œuvre barthésienne cède lentement la place dans ses travaux des années 1970 au désir du neutre. Le dernier Barthes - de plus en plus fatigué par son corps, par se migraines et ses jouissances -, se lance dans le rêve du neutre, d'une force qui ne procède pas au classement, à la division, à l'inévitable structuration :

Il a parfois envie de laisser reposer tout ce langage qui est dans sa tête, dans son travail, dans les autres, comme si le langage était lui-même un membre fatigué du corps humain ; il lui semble que s'il se reposait du langage, il se reposerait tout entier, par congé donné aux crises, aux retentissements, aux exaltations, aux blessures, aux raisons, etc. (Barthes, 1975, p. 179).

Sans entrer dans l'explication du neutre, retenons seulement qu'il est une pratique consistant à s'affranchir du battement binaire de la signification et à saisir ce qui échappe au sens engendré par une telle structuration. " "Déjouer le paradigme" est une activité ardente, brûlante » (Barthes, 2002, p. 32). Le Neutre, c'est comme il écrit : « tout ce qui esquive ou déjou ou rend dérisoires la parade, la maîtrise, l'intimidation » (Barthes, 1975, p. 136). Le neutre est-il donc possibilité d'accéder à l'état archaïque ou présémantique du corps, à son immédiateté qui bouleverse toutes les formes ? Les Cours sur le neutre prononcés au Collège de France sont en fait une ébauche de la pensée qui n'a pas eu le temps de prendre une forme définitive.

\section{EXISTENCE SÉMIOTIQUE}

Désormais il est clair que l'expérience sensuelle n'est pas immédiate, elle est en rapport étroit avec l'intellect, l'intelligence, la pensée. Écouter, voir, sentir n'est jamais pur et inversement le langage porte toujours des traces du sentir. L'expérience de la sensation passe par le moi qui n'arrête pas de s'observer, d'analyser ses percep- 
tions, de les nommer, voire de diviser, la parole est toujours remué par les pulsions et les frémissements du corps. Or ces constations semblent aujourd'hui plus qu'évidentes après tant d'années des recherches sémiotiques. La sémiotique des passions de Algirdas-Julien Greimas et de Jean Fontanille l'éclaire admirablement. Cependant les intuitions de Barthes ont devancé certaines de leurs conclusions ainsi que de multiples modes dont nous sommes prisonniers.

Mais il reste toujours des interrogations sur le fondement de la conception sémiotique du corps barthésien. Le moi perceptif relate la façon dont il vit son propre corps et le monde. Le moi est au carrefour du corps et du signe. Comment peuton percevoir son corps sans recourir au signe ? Peut-on sortir de l'opacité du corps, peut-on éviter sa médiation? S'agit-il de retourner à l'état préréflexif à la manière de Merleau-Ponty ? Pourrait-on y voir un accent phénoménologique dans la démarche barthésienne ? Oui et non. Les signes ne sont pas seulement un " paraître » chez Barthes, le monde, le corps et les signes sont déjà là, en interaction.

L'effort de Barthes est d'interroger constamment la frontière qui sépare le réel et l'imaginaire, le corps et le signe, la nature et l'histoire, l'ipséité de sa propre expérience et son altérité sémiotique.

\section{THÉÂTRE DU CORPS}

L'écrivain est donc celui à qui le corps fait problème. Le corps tant qu'il est vivant, il est toujours enchaîné dans le système des signes. Tant qu'il est vivant, son devenir est textuel, sémiotique. Ivan Almeida parlait d'un « corps devenu récit ». Pour Barthes le corps est un personnage du roman, il n'est pas un héros. Toujours en position passive, réceptive, il a pourtant la faculté de moduler des fictions qu'il doit jouer. Barthes reste un observateur attentif des expériences et des sensations dont il est objet en tant qu'être charnel. Malgré sa méfiance au langage, le sentir doit être toujours nommé pour sauver le corps et soi-même de l'asymbolie, du silence auquel il risquerait d'être réduit. Barthes reste très retenu à l'égard de la mort. Sans hystérie il parle de la disparition du corps, produite par le mauvais choix d'un signe ou par l'absence de la nomination comme s'il gardait en mémoire la phrase de Bossuet :

Que vous servira d'avoir tant écrit dans ce livre, d'en avoir rempli toutes les pages de beaux caractères, puisque enfin une seule rature doit tout effacer ? Encore une rature laisserait-elle quelques traces du moins d'elle-même ; au lieu que ce dernier moment qui effacera d'un seul trait toute votre vie, s'ira perdre lui-même, avec tout le reste, dans ce grand gouffre du néant. Il n'y aura plus sur la terre aucun vestige de ce que nous sommes : la chair changera de nature : le corps prendra un autre nom ; même celui de cadavre ne lui demeurera pas longtemps : il deviendra, dit Tertullien, un je ne sais quoi qui n'a plus de nom dans aucune langue (Bossuet, Sermon sur la mort, 1662). 
Sauver le corps de l'indicible, du mutisme, mais aussi de l'aliénation à laquelle le porte le langage, la rhétorique et l'Institution, c'était peut-être l'enjeu qu'il affrontait et c'était peut-être le combat qu'il menait avec les signes, combat acharné et éprouvant qui l'a conduit à la tentation du non-vouloir-saisir.

\section{BIBLIOGRAPHIE}

Almeida, I. (1983). Un corps devenu récit. Dans C. Reichler, Le corps et ses fictions (pp. 7-18). Paris : Les Éditions de Minuit.

Barthes, R. (1953 et 1972). Le degré zéro de l'écriture suivi de Nouveaux essais critiques. Paris : Seuil.

- (1973). Le Plaisir du texte. Paris : Seuil.

- (1975). Roland Barthes par Roland Barthes. Paris : Seuil.

- (1977). Fragments d'un discours amoureux. Paris : Seuil.

- (1982). Le grain de la voix. Dans R. Barthes, L'obvie et l'obtus. Essais critiques III (pp. 236-245).

Paris : Seuil.

- (1987). Incidents. Paris : Seuil.

- (2002). Le Neutre. Cours au Collège de France (1977-1978). Paris : Seuil.

- (2006). Roland Barthes, le métier d'écrire. Paris : Seuil. 